\title{
Axiomatizations of Banzhaf permission values for games with a permission structure
}

\author{
René van den Brink
}

Accepted: 15 December 2009 / Published online: 22 January 2010

(C) The Author(s) 2010. This article is published with open access at Springerlink.com

\begin{abstract}
In games with a permission structure it is assumed that players in a cooperative transferable utility game are hierarchically ordered in the sense that there are players that need permission from other players before they are allowed to cooperate. We provide axiomatic characterizations of Banzhaf permission values being solutions that are obtained by applying the Banzhaf value to modified TU-games. In these characterizations we use power- and player split neutrality properties. These properties state that splitting a player's authority and/or contribution over two players does not change the sum of their payoffs.
\end{abstract}

Keywords Cooperative game theory - Permission structure $\cdot$ Banzhaf value Split neutrality

\section{JEL classification $\mathrm{C} 71 \cdot \mathrm{Z} 13$}

\section{Introduction}

A situation in which a finite set of players $N \subset \mathbb{N}$ can generate certain payoffs by cooperation can be described by a cooperative game with transferable utility (or simply a TU-game), being a pair $(N, v)$ where $v: 2^{N} \rightarrow \mathbb{R}$ is a characteristic function on

\footnotetext{
A preliminary version of this article is published in the ICM Millennium Lectures on Games (eds. L.A. Petrosyan and D.W.K. Yeung), Springer-Verlag, Heidelberg, Germany (2003), without permission from the author.
}

R. van den Brink $(\varangle)$

Department of Econometrics and Tinbergen Institute, VU University,

De Boelelaan 1105, 1081 HV Amsterdam, The Netherlands

e-mail: jrbrink@feweb.vu.nl 
$N$ satisfying $v(\emptyset)=0$. For any coalition $E \subseteq N, v(E) \in \mathbb{R}$ is the worth of coalition $E$, i.e. the members of coalition $E$ can obtain a total payoff of $v(E)$ by agreeing to cooperate. We denote the collection of all characteristic functions on player set $N$ by $\mathcal{G}^{N}$.

In a TU-game $(N, v)$ there are no restrictions on the cooperation possibilities of the players, i.e. every coalition $E \subseteq N$ is feasible and can generate a worth. Various models with restrictions on coalition formation are discussed in the literature. In this paper we consider games with a permission structure which describe situations in which the players in a TU-game are part of a hierarchical organization, referred to as a permission structure, such that there are players that need permission from other players before they are allowed to cooperate. ${ }^{1}$ Thus, the possibilities of coalition formation are determined by the positions of the players in the permission structure. Various assumptions can be made about how a permission structure affects the cooperation possibilities. In the disjunctive approach as considered in Gilles and Owen (1999) and van den Brink (1997) it is assumed that every player needs permission from at least one of its predecessors before it is allowed to cooperate with other players. Alternatively, in the conjunctive approach as developed in Gilles et al. (1992) and van den Brink and Gilles (1996), it is assumed that every player needs permission from all its predecessors before it is allowed to cooperate.

To take account of the limited cooperation possibilities, for every game with a permission structure a modified game is defined which assigns to every coalition the worth of its largest feasible subcoalition in the original game. The disjunctive and conjunctive approach yield different modified games. A solution for games with a permission structure is a function that assigns to every such a game a payoff distribution over the individual players. Applying solutions for TU-games to the modified games yields solutions for games with a permission structure. In the literature axiomatizations can be found of the disjunctive and conjunctive Shapley permission values, which are obtained by applying the Shapley value (1953) to the disjunctive, respectively conjunctive, modified game. The underlying paper considers the disjunctive and conjunctive Banzhaf permission values, which are obtained by applying the Banzhaf value (which is based on the Banzhaf index for voting games (1965) and is generalized to TU-games by, e.g., Owen (1975) and Dubey and Shapley (1979)) to the two modified games.

In van den Brink $(1997,1999)$ it is shown that the difference between the two Shapley permission values boils down to applying a different fairness or equal gain/loss property. Disjunctive fairness implies that deleting the arc between a predecessor and one of its successors (who has at least one other predecessor) changes the payoffs of these two players by the same amount. Alternatively, conjunctive fairness implies that deleting the arc between a predecessor and a successor changes the payoffs of that successor and any of its other predecessors by the same amount. It is easy to verify that the disjunctive, respectively conjunctive, Banzhaf permission values also satisfy disjunctive, respectively conjunctive, fairness.

\footnotetext{
1 Examples of other models where there are restrictions on the possibilities of cooperation are the games in coalition structure, see, e.g., Aumann and Drèze (1974) and games with limited communication structure, see, e.g. Myerson (1977).
} 
These two fairness properties compare the effects of deleting the arc between two players on the payoffs of these two players, respectively, on the payoffs of the successor and its other predecessors. They do not compare the change in payoffs of the predecessor of the deleted arc and other predecessors of the successor. Although the effect of deleting an arc on the payoffs of players depends on the game, it seems reasonable that for any game the change in payoffs of the predecessor and any other predecessor of the successor on the deleted arc are in opposite direction. It turns out that the Shapley permission values do not satisfy this 'opposite change' property. However, it turns out that the Banzhaf permission values do satisfy this property. They even satisfy the stronger property that the sum of the payoffs of the two predecessors does not change. Since creating an arc between a player and a predecessor can be seen as a split of power over the successor between its predecessors, we refer to this property as power split neutrality.

The main result of this paper is to give an axiomatic characterization of the two Banzhaf permission values using this power split neutrality property. In order to do that we need to add two more new axioms that both represent a split of player's positions in the permission structure and/or contributions in the game. Here we can extend split or merge neutrality properties that are similar to those used to characterize the Banzhaf value for TU-games in, e.g. Lehrer (1988) and Haller (1994). It turns out that these properties can be adapted in an intuitive way to games with a permission structure where we consider splitting player's positions in the permission structure and their contributions in the game. We introduce two player split neutrality properties stating that, if a player in a game with permission structure 'splits' in two player's then the sum of payoffs of the two players into which the existing player is split is equal to the payoff of the already existing player in the original game with permission structure. This split concerns both the contributions of the players in the game as well as their positions in the permission structure. With respect to these positions we distinguish between a vertical and a horizontal split of a player.

We provide axiomatizations of the Banzhaf permission values using power- and both player split neutrality properties. Similar as with the Shapley permission values, the difference between the disjunctive- and conjunctive Banzhaf permission values is with respect to the fairness axiom.

The paper is organized as follows. In Sect. 2 we state some preliminaries on games with a permission structure. In Sect. 3 we introduce the player- and power split neutrality properties. In Sect. 4 we then provide axiomatic characterizations of the Banzhaf permission values, and show logical independence of the axioms. In Sect. 5 we make some concluding remarks, including adaptations of the split neutrality properties to obtain new axiomatic characterizations of the Shapley permission values.

\section{Preliminaries: games with a permission structure}

A permission structure on a finite set of players $N \subset \mathbb{N}$ is a mapping $S: N \rightarrow 2^{N}$. The players in $S(i)$ are called the successors of player $i \in N .^{2}$ The players in $S^{-1}(i):=$

\footnotetext{
${ }^{2}$ Note that the set $\{(i, j) \in N \times N \mid i \in N, j \in S(i)\}$ describes a directed graph on $N$.
} 
$\{j \in N \mid i \in S(j)\}$ are called the predecessors of $i$. A pair $(i, j) \in N \times N$ with $j \in S(i)$ is called an arc in $S$. By $\widehat{S}$ we denote the transitive closure of the permission structure $S$, i.e., $j \in \widehat{S}(i)$ if and only if there exists a sequence of players $\left(h_{1}, \ldots, h_{t}\right)$ such that $h_{1}=i, h_{k+1} \in S\left(h_{k}\right)$ for all $1 \leq k \leq t-1$, and $h_{t}=j$. The players in $\widehat{S}(i)$ are called the subordinates of $i$, and the players in $\widehat{S}^{-1}(i):=\{j \in N \mid i \in \widehat{S}(j)\}$ are called the superiors of $i$. In this paper we restrict our attention to hierarchical permission structures being permission structures $S: N \rightarrow 2^{N}$ that are

(i) acyclic, i.e., $i \notin \widehat{S}(i)$ for all $i \in N$, and

(ii) quasi-strongly connected, i.e., there exists an $i \in N$ such that $\widehat{S}(i)=N \backslash\{i\}$.

In a hierarchical permission structure there exists a unique player $i_{0}$ such that $\widehat{S}\left(i_{0}\right)=N \backslash\left\{i_{0}\right\}$. Moreover, $S^{-1}\left(i_{0}\right)=\emptyset$ for this player. We call this player the topplayer in the permission structure. Examples of hierarchical permission structures are permission trees in which every player, except the top-player, has exactly one predecessor. Since we only consider hierarchical permission structures we will often refer to these simply as permission structures. We denote the collection of all (hierarchical) permission structures on a particular player set $N$ by $\mathcal{S}^{N}$. A triple $(N, v, S)$ with $N \subset \mathbb{N}, v \in \mathcal{G}^{N}$ and $S \in \mathcal{S}^{N}$ is called a game with a (hierarchical) permission structure.

In the disjunctive approach to games with a permission structure as developed in Gilles and Owen (1999) and van den Brink (1997), it is assumed that each player needs permission from at least one of its predecessors before it is allowed to cooperate with other players. Consequently, a coalition is feasible if and only if every player in the coalition, except the top-player $i_{0}$, has at least one predecessor who also belongs to the coalition. Thus, the feasible coalitions are the ones in the set

$$
\Phi_{N, S}^{d}:=\left\{E \subseteq N \mid S^{-1}(i) \cap E \neq \emptyset \text { for all } i \in E \backslash\left\{i_{0}\right\}\right\} .
$$

Note that this implies that the top-player $i_{0}$ belongs to every nonempty coalition in $\Phi_{N, S}^{d}$. The coalitions in $\Phi_{N, S}^{d}$ are called the disjunctive autonomous coalitions in $S$. The largest disjunctive autonomous subset of $E \subseteq N$ in $S \in \mathcal{S}^{N}$ is denoted by $\sigma_{N, S}^{d}(E)=\cup\left\{F \in \Phi_{N, S}^{d} \mid F \subseteq E\right\}$, and is called the disjunctive sovereign part of $E$ in $S .{ }^{3}$ It consists of those players in $E$ that can be reached by a directed 'permission path' starting from the top-player such that all players on this path belong to coalition $E$. Given a game with a permission structure $(N, v, S)$, the disjunctive restriction of $v$ on $S$ is the modified characteristic function $r_{N, v, S}^{d}: 2^{N} \rightarrow \mathbb{R}$ which assigns to every coalition the worth of its largest disjunctive autonomous subcoalition and thus is given by $r_{N, v, S}^{d}(E)=v\left(\sigma_{N, S}^{d}(E)\right)$ for all $E \subseteq N$.

Alternatively, in the conjunctive approach as developed in Gilles et al. (1992), van den Brink and Gilles (1996) and van den Brink (1999), it is assumed that each player needs permission from all its predecessors before it is allowed to cooperate. This means that a coalition $E$ is feasible if and only if for every player in the coalition it

\footnotetext{
${ }^{3}$ By the set of disjunctive autonomous coalitions being closed under union, these largest feasible subsets exist and are unique. Note the difference with the modified games of Myerson (1977) for games with limited communication structure, which assign to every coalition the sum of the worths of its components.
} 
Fig. 1 Permission structure $S$ of Example 2.1

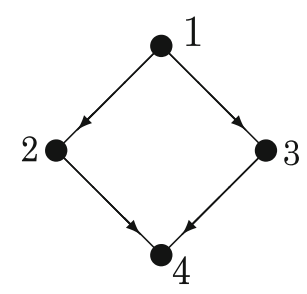

holds that all its predecessors belong to the coalition. The set of feasible coalitions in this approach thus is given by

$$
\Phi_{N, S}^{c}:=\left\{E \subseteq N \mid S^{-1}(i) \subseteq E \text { for all } i \in E\right\}
$$

The coalitions in the set $\Phi_{N, S}^{c}$ are called the conjunctive autonomous coalitions in $S$. The largest conjunctive autonomous subset of $E$ is denoted by $\sigma_{N, S}^{c}(E)=\cup\{F \in$ $\Phi_{N, S}^{c} \mid F \subseteq E$, and is referred to as the conjunctive sovereign part of $E$ in $S$. It consists of all players in $E$ whose predecessors all belong to $E$. Given game with permission structure $(N, v, S)$, the conjunctive restriction of $v$ on $S$ is the characteristic function $r_{N, v, S}^{c}: 2^{N} \rightarrow \mathbb{R}$ given by $r_{N, v, S}^{c}(E)=v\left(\sigma_{N, S}^{c}(E)\right)$ for all $E \subseteq N$.

Example 2.1 Consider the game with permission structure $(N, v, S)$ with $N=$ $\{1,2,3,4\}, v \in \mathcal{G}^{N}$ given by $v(E)=1$ if $4 \in E, v(E)=0$ if $4 \notin E$, and $S \in \mathcal{S}^{N}$ given by $S(1)=\{2,3\}, S(2)=S(3)=\{4\}, S(4)=\emptyset$ (Fig. 1).

The disjunctive restriction of $v$ on $S$ is given by $r_{N, v, S}^{d}(E)=1$ if $E \in\{\{1,2,4\}$, $\{1,3,4\},\{1,2,3,4\}\}$, and $r_{N, v, S}^{d}(E)=0$ otherwise. The conjunctive restriction of $v$ on $S$ is given by $r_{N, v, S}^{c}(E)=1$ if $E=\{1,2,3,4\}$, and $r_{N, v, S}^{c}(E)=0$ otherwise.

A solution for games with a permission structure is a function $f$ that assigns a payoff distribution $f(N, v, S) \in \mathbb{R}^{N}$ to every game with permission structure $(N, v, S)$. The disjunctive Banzhaf permission value $\beta^{d}$ is obtained by applying the Banzhaf value to the disjunctive restricted game, while the conjunctive Banzhaf permission value $\beta^{c}$ is obtained by applying the Banzhaf value to the conjunctive restricted game, i.e.,

$$
\beta^{d}(N, v, S)=B a\left(N, r_{N, v, S}^{d}\right) \text { and } \beta^{c}(N, v, S)=B a\left(N, r_{N, v, S}^{c}\right),
$$

where

$$
B a_{i}(N, v)=\frac{1}{2^{|N|-1}} \sum_{\substack{E \subseteq N \\ i \in E}}(v(E)-v(E \backslash\{i\})) \text { for all } i \in N
$$

Alternatively, applying the Shapley value to the disjunctive- and conjunctive restricted games yields, respectively, the disjunctive Shapley permission value $\varphi^{d}$ and the conjunctive Shapley permission value $\varphi^{c}$, i.e.,

$$
\varphi^{d}(N, v, S)=\operatorname{Sh}\left(N, r_{N, v, S}^{d}\right) \quad \text { and } \varphi^{c}(N, v, S)=\operatorname{Sh}\left(N, r_{N, v, S}^{c}\right)
$$


where

$$
\operatorname{Sh}_{i}(N, v)=\sum_{\substack{E \subseteq N \\ i \in E}} \frac{(|N|-|E|) !(|E|-1) !}{|N| !}(v(E)-v(E \backslash\{i\})) \text { for all } i \in N
$$

Example 2.2 The disjunctive- and conjunctive Banzhaf permission values of the game with permission structure given in Example 2.1 are $\beta^{d}(N, v, S)=\frac{1}{8}(3,1,1,3)$ and $\beta^{c}(N, v, S)=\frac{1}{8}(1,1,1,1)$. The disjunctive- and conjunctive Shapley permission values are $\varphi^{d}(N, v, S)=\frac{1}{12}(5,1,1,5)$ and $\varphi^{c}(N, v, S)=\frac{1}{4}(1,1,1,1)$.

Player $i \in N$ is inessential in game with permission structure $(N, v, S)$ if $i$ and all its subordinates are null players in $(N, v)$, i.e., if $v(E)=v(E \backslash\{j\})$ for all $E \subseteq N$ and $j \in\{i\} \cup \widehat{S}(i)$. Player $i \in N$ is called necessary in game $(N, v)$ if $v(E)=0$ for all $E \subseteq N \backslash\{i\}$. We say that player $i \in N$ dominates player $j \in N$ completely if all directed 'permission paths' from the top-player $i_{0}$ to player $j$ contain player $i$. We denote the set of players that player $i$ dominates completely by $\bar{S}(i)$, i.e.,

$$
\begin{aligned}
& \bar{S}(i) \\
& \quad=\left\{j \in \widehat{S}(i) \mid \begin{array}{c}
i \in\left\{h_{1}, \ldots, h_{t-1}\right\} \text { for every sequence of nodes } h_{1}, \ldots, h_{t} \\
\text { such that } h_{1}=i_{0}, h_{k+1} \in S\left(h_{k}\right), k \in\{1, \ldots, t-1\}, \quad \text { and } h_{t}=j
\end{array}\right\} .
\end{aligned}
$$

We also define $\bar{S}^{-1}(i)=\left\{j \in \widehat{S}^{-1}(i) \mid i \in \bar{S}(j)\right\}$. A characteristic function $v \in \mathcal{G}^{N}$ is monotone if $v(E) \leqq v(F)$ for all $E \subseteq F \subseteq N$. The class of all monotone characteristic functions on $N$ is denoted by $\mathcal{G}_{M}^{N}$. Next we recall some axiomatizations of the Shapley permission values. ${ }^{4}$

Efficiency For every $N \subset \mathbb{N}, v \in \mathcal{G}^{N}$ and $S \in \mathcal{S}^{N}$, it holds that $\sum_{i \in N} f_{i}(N, v, S)=$ $v(N)$.

Additivity For every $N \subset \mathbb{N}, v, w \in \mathcal{G}^{N}$ and $S \in \mathcal{S}^{N}$, it holds that $f(N, v+$ $w, S)=f(N, v, S)+f(N, w, S)$, where $(v+w) \in \mathcal{G}^{N}$ is given by $(v+w)(E)=$ $v(E)+w(E)$ for all $E \subseteq N$.

Inessential player property For every $N \subset \mathbb{N}, v \in \mathcal{G}^{N}$ and $S \in \mathcal{S}^{N}$, if $i \in N$ is an inessential player in $(N, v, S)$ then $f_{i}(N, v, S)=0$.

Necessary player property For every $N \subset \mathbb{N}, v \in \mathcal{G}_{M}^{N}$ and $S \in \mathcal{S}^{N}$, if $i \in N$ is a necessary player in $(N, v)$ then $f_{i}(N, v, S) \geqq f_{j}(N, v, S)$ for all $j \in N$.

Weak structural monotonicity For every $N \subset \mathbb{N}, v \in \mathcal{G}_{M}^{N}$ and $S \in \mathcal{S}^{N}$, if $i \in N$ and $j \in \bar{S}(i)$ then $f_{i}(N, v, S) \geq f_{j}(N, v, S)$.

Both Shapley permission values satisfy these five axioms. Further, the disjunctive Shapley permission value satisfies disjunctive fairness which states that deleting the arc between two players $h$ and $j \in S(h)$ (with $\left|S^{-1}(j)\right| \geq 2$ ) changes the payoffs of players $h$ and $j$ by the same amount. Moreover, also the payoffs of all players $i$ that

4 We refer to van den Brink and Gilles (1996) and van den Brink (1997) for a discussion of these properties. 
completely dominate player $h$ change by this same amount. ${ }^{5}$ It can be verified from Examples 2.1 and 2.2 that the conjunctive Shapley permission value does not satisfy disjunctive fairness. However, it satisfies the alternative conjunctive fairness which states that deleting the arc between two players $h$ and $j \in S(h)$ (with $\left|S^{-1}(j)\right| \geq 2$ ) changes the payoffs of player $j$ and any other predecessor $g \in S^{-1}(j) \backslash\{h\}$ by the same amount. Moreover, also the payoffs of all players that completely dominate the other predecessor $g$ change by this same amount. For $S \in \mathcal{S}^{N}, h \in N$ and $j \in S(h)$ we denote the permission structure that is left after deleting the arc between $h$ and $j$ by

$$
S_{-(h, j)}(i)= \begin{cases}S(i) \backslash\{j\} & \text { if } i=h \\ S(i) & \text { if } i \in N \backslash\{h\} .\end{cases}
$$

Disjunctive fairness For every $N \subset \mathbb{N}, v \in \mathcal{G}^{N}$ and $S \in \mathcal{S}^{N}$, if $h \in N$ and $j \in$ $S(h)$ with $\left|S^{-1}(j)\right| \geq 2$ then $f_{j}(N, v, S)-f_{j}\left(N, v, S_{-(h, j)}\right)=f_{i}(N, v, S)-$ $f_{i}\left(N, v, S_{-(h, j)}\right)$ for all $i \in\{h\} \cup \bar{S}^{-1}(h)$.

Conjunctive fairness For every $N \subset \mathbb{N}, v \in \mathcal{G}^{N}$ and $S \in \mathcal{S}^{N}$, if $h, j, g \in N$ are such that $h \neq g$ and $j \in S(h) \cap S(g)$ then $f_{j}(N, v, S)-f_{j}\left(N, v, S_{-(h, j)}\right)=$ $f_{i}(N, v, S)-f_{i}\left(N, v, S_{-(h, j)}\right)$ for all $i \in\{g\} \cup \bar{S}^{-1}(g)$.

The axioms described above characterize the two Shapley permission values.

Theorem 2.3 (i) (van den Brink 1997) A solution $f$ is equal to the disjunctive Shapley permission value $\varphi^{d}$ if and only if it satisfies efficiency, additivity, the inessential player property, the necessary player property, weak structural monotonicity and disjunctive fairness.

(ii) (van den Brink 1999) A solution $f$ is equal to the conjunctive Shapley permission value $\varphi^{c}$ if and only if it satisfies efficiency, additivity, the inessential player property, the necessary player property, weak structural monotonicity and conjunctive fairness.

\section{Power and player split neutrality}

The two fairness axioms that are mentioned in the previous section compare the effects of deleting the arc between players $h$ and $j \in S(h)$ on the payoffs of players $h$ and $j$, respectively, on the payoffs of players $g \in S^{-1}(j) \backslash\{h\}$ and $j$. These properties do not compare the change in payoffs of players $h$ and $g \in S^{-1}(j) \backslash\{h\}$ after deleting the arc between $h$ and $j$. The opposite change property states that deleting the arc between player $h$ and $j \in S(h)$ (with $\left|S^{-1}(j)\right| \geq 2$ ) changes the payoffs of players $h$ and $g \in S^{-1}(j) \backslash\{h\}$ in opposite direction. The Shapley permission values do not

\footnotetext{
5 This property is some kind of equal-loss-or-gain property. Since it is related to fairness as introduced in Myerson (1977) for games with a limited communication structure, we refer to this property as (disjunctive) fairness. Note that in disjunctive fairness we require that the successor on the arc to be deleted has at least two predecessors, implying that the permission structure that is left after deleting the arc is still quasi-strongly connected.
} 
Fig. 2 An illustration of permission structures $S$ and $S^{V(h, j)}$
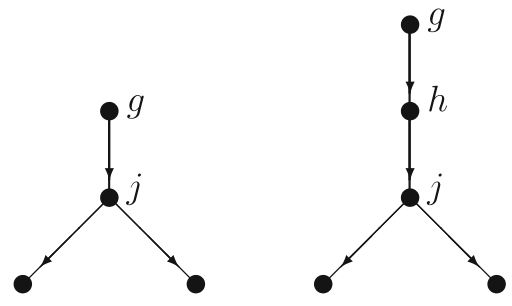

satisfy this property. ${ }^{6}$ It turns out that the disjunctive and conjunctive Banzhaf permission values do satisfy this opposite change property. They even satisfy the stronger property which requires that the sum of the payoffs of the two predecessors does not change. Since creating an arc between a player and a predecessor can be seen as a split of the power over this player (i.e. a transfer of the authority of $h$ over $j$ to player $g$ ), we refer to this property as power split neutrality.

Power split neutrality For every $N \subset \mathbb{N}, v \in \mathcal{G}^{N}$ and $S \in \mathcal{S}^{N}$, if $h, g, j \in N$ are such that $h \neq g$ and $j \in S(h) \cap S(g)$ then $f_{h}(N, v, S)+f_{g}(N, v, S)=$ $f_{h}\left(N, v, S_{-(h, j)}\right)+f_{g}\left(N, v, S_{-(h, j)}\right)$.

In the context of (unrestricted) TU-games, Haller (1994) uses collusion neutrality properties in characterizing the Banzhaf value. Collusion neutrality properties are some kind of stability properties in the sense that no two players can do better by merging into one player, and no single player does better by splitting in two. ${ }^{7}$ Whereas Haller (1994)'s collusion properties concern the collusion of players contributions in the game, power split neutrality as defined above can be seen as a collusion of players with respect to their authority in the permission structure, keeping the game unchanged. In terms of games with a permission structure, Haller (1994)'s collusion neutrality properties with respect to player contributions can be nicely interpreted in terms of the game and the permission structure, where both a player's contribution in the game as well as its position in the permission structure is split. We consider two such properties: one in which a player splits in two players in a vertical line, and another in which a player splits at the bottom level. ${ }^{8}$

First, let $h \in \mathbb{N} \backslash N$ be a new player whose only task is to supervise player $j$. So, $h$ will be added as a null player to the game, and in the permission structure he becomes the only predecessor of $j$ and gets all previous predecessors of $j$ as its predecessors, see Fig. 2. Since its only task is to supervise player $j$, the new player $h$ does not really

\footnotetext{
${ }^{6}$ Consider, for example, the game with permission structure $(N, v, S)$ given by $N=\{1,2,3,4,5\}$, $v=u_{\{4,5\}}-\frac{7}{10} u_{\{4\}}$, where $u_{T}$ denotes the unanimity game of $T \subseteq N, T \neq \varnothing$ [see (5) with $c_{T}$ $=1], S(1)=\{2,3,5\}, S(2)=S(3)=\{4\}$ and $S(4)=S(5)=\emptyset$. In this example, $\varphi_{2}^{d}(N, v, S)-$ $\varphi_{2}^{d}\left(N, v, S_{-(2,4)}\right)=-\frac{1}{120}$ and $\varphi_{3}^{d}(N, v, S)-\varphi_{3}^{d}\left(N, v, S_{-(2,4)}\right)=-\frac{1}{40}$. For the conjunctive Shapley permission values $\varphi_{2}^{c}(N, v, S)-\varphi_{2}^{c}\left(N, v, S_{-(2,4)}\right)=\frac{1}{40}$ and $\varphi_{3}^{c}(N, v, S)-\varphi_{3}^{c}\left(N, v, S_{-(2,4)}\right)=\frac{1}{120}$.

7 We refer to Haller (1994) for further motivation of such neutrality properties.

8 The vertical and horizontal split neutrality axioms used here are based on a slightly different neutrality property for TU-games as that of Haller (1994) and is defined in the appendix of this paper. Other neutrality axioms for TU-games are introduced in Lehrer (1988).
} 
Fig. 3 An illustration of permission structures $S$ and $S^{H(h, j)}$
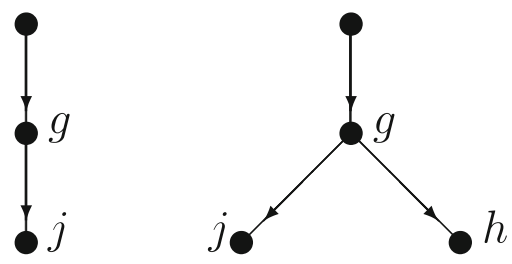

add anything to the generation of value that was not yet generated by player $j$, and therefore we argue that after such a vertical split the sum of the payoffs of players $h$ and $j$ in the new game with permission structure should be the same as the payoff of player $j$ in the original game with permission structure.

Vertical split neutrality For every $N \subset \mathbb{N}, v \in \mathcal{G}^{N}$ and $S \in \mathcal{S}^{N}$, if $h \in \mathbb{N} \backslash N$ and $j \in N$ then $f_{j}\left(N \cup\{h\}, v^{V(h, j)}, S^{V(h, j)}\right)+f_{h}\left(N \cup\{h\}, v^{V(h, j)}, S^{V(h, j)}\right)=$ $f_{j}(N, v, S)$, where $v^{V(h, j)} \in \mathcal{G}^{N \cup\{h\}}$ is given by

$$
v^{V(h, j)}(E)=v(E \cap N) \text { for all } E \subseteq N \cup\{h\},
$$

and $S^{V(h, j)} \in \mathcal{S}^{N \cup\{h\}}$ is given by

$$
S^{V(h, j)}(i)= \begin{cases}\{j\} & \text { if } i=h \\ (S(i) \backslash\{j\}) \cup\{h\} & \text { if } i \in S^{-1}(j) \\ S(i) & \text { if } i \in N \backslash S^{-1}(j)\end{cases}
$$

For the second player split neutrality property, suppose that 'bottom' player $j \in N$ (having no successors), is split in two players at the same level in the sense that a new player $h$ enters who gets the same predecessors as $j$, has no successors (see Fig. 3), while in the new game $h$ and $j$ veto each other. Again, the presence of $h$ does not essentially change the generation of value, except that $h$ is needed to get the productive participation of $j$. Similar as above, we argue that the sum of the payoffs of players $h$ and $j$ in the new game with permission structure should be equal to the payoff of player $j$ in the original game with permission structure.

Horizontal split neutrality For every $N \subset \mathbb{N}, v \in \mathcal{G}^{N}$ and $S \in \mathcal{S}^{N}$, if $h \in \mathbb{N} \backslash N$ and $j \in N$ is such that $S(j)=\varnothing$ and $S^{-1}(j) \neq \varnothing$ then $f_{j}\left(N \cup\{h\}, v^{H(h, j)}, S^{H(h, j)}\right)+f_{h}\left(N \cup\{h\}, v^{H(h, j)}, S^{H(h, j)}\right)=f_{j}(N, v, S)$, where $v^{H(h, j)} \in \mathcal{G}^{N \cup\{h\}}$ is given by

$$
v^{H(h, j)}(E)= \begin{cases}v(E \backslash\{j\}) & \text { if } E \subseteq N \\ v(E \cap N) & \text { if } E \subseteq N \cup\{h\} \text { with } h \in E,\end{cases}
$$

and $S^{H(h, j)} \in \mathcal{S}^{N \cup\{h\}}$ is given by

$$
S^{H(h, j)}(i)= \begin{cases}\emptyset & \text { if } i=h \\ S(i) \cup\{h\} & \text { if } i \in S^{-1}(j) \\ S(i) & \text { if } i \in N \backslash S^{-1}(j) .\end{cases}
$$


Proposition 3.1 The disjunctive- and conjunctive Banzhaf permission values satisfy power split neutrality, vertical split neutrality and horizontal split neutrality.

Proof The proof that $\beta^{d}$ satisfies power split neutrality is straightforward as soon as one realizes that $\sigma_{N, S}^{d}(E)=\sigma_{N, S_{-(h, j)}}^{d}(E)$ whenever $h \notin E$ or $g \in E$. The proof that $\beta^{c}$ satisfies power split neutrality is straightforward as soon as one realizes that $\sigma_{N, S}^{c}(E)=\sigma_{N, S_{-(h, j)}}^{c}(E)$ if $h \in E$ or $g \notin E$.

Vertical split neutrality of $\beta^{d}$ follows from Proposition A.1 in the appendix and the fact that $r_{N, v^{V(h, j)}, S^{V(h, j)}}^{d}=\left(r_{N, v, S}^{d}\right)_{h j}$ where $v_{h j}$ is as given in (16). ${ }^{9}$ Horizontal split neutrality of $\beta^{d}$ follows from Proposition A.1 and the fact that $r_{N, v^{H(h, j)}, S^{H(h, j)}}^{d}=$ $\left(r_{N, v, S}^{d}\right)_{h j}$. Similar for $\beta^{c}$.

\section{Axiomatizations of Banzhaf permission values}

From the axioms that characterize the Shapley permission values in Theorem 2.3, the Banzhaf permission values do not satisfy efficiency. However, they satisfy the weaker axiom which requires efficiency only for one-player games.

One-player efficiency For every $i \in \mathbb{N}, v \in \mathcal{G}^{\{i\}}$ and $S \in \mathcal{S}^{\{i\}}$, it holds that $f_{i}(\{i\}, v, S)=v(\{i\})$.

Together with the power and player split neutrality axioms of the previous section and the other properties that characterized the Shapley permission values in Theorem 2.3, this yields axiomatizations of the Banzhaf permission values. Similar as with the Shapley permission values the two Banzhaf permission values differ with respect to the fairness axiom.

Theorem 4.1 (i) A solution $f$ is equal to the disjunctive Banzhaf permission value $\beta^{d}$ if and only if it satisfies power split neutrality, vertical split neutrality, horizontal split neutrality, one-player efficiency, additivity, the inessential player property, the necessary player property, weak structural monotonicity and disjunctive fairness.

(ii) A solution $f$ is equal to the conjunctive Banzhaf permission value $\beta^{c}$ if and only if it satisfies power split neutrality, vertical split neutrality, horizontal split neutrality, one-player efficiency, additivity, the inessential player property, the necessary player property, weak structural monotonicity and conjunctive fairness.

Necessity of the first three axioms follows from Proposition 3.1. Proving that the Banzhaf permission values satisfy additivity, the inessential player property, the necessary player property, weak structural monotonicity and disjunctive, respectively conjunctive, fairness is along the same lines as this is shown for the Shapley permission values in van den Brink (1997) for $\varphi^{d}$ and in van den Brink and Gilles (1996) and

\footnotetext{
9 These statements also follow from Theorem 1 in Algaba et al. (2004) after showing that the modified antimatroids in that paper are equal to the set of disjunctive feasible coalitions in the manipulated permission structure.
} 
Fig. 4 An illustration of permission structure $S_{-h}$
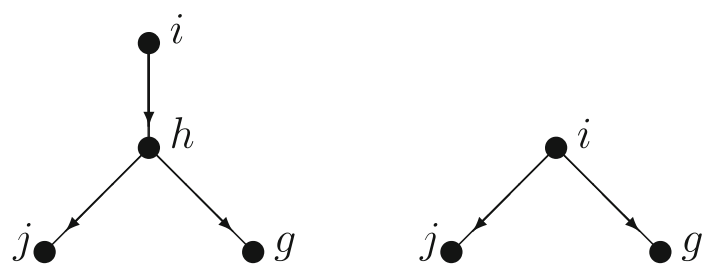

van den Brink (1999) for $\varphi^{c}$. Since one-player efficiency is obvious, we focus on the uniqueness part of the proof. We prove the uniqueness part of Theorem 4.1.(i) in three steps. First, we prove a lemma for positively scaled unanimity games with a permission tree that has no inessential players. We denote by $\mathcal{S}_{\text {tree }}^{N}=\left\{S \in \mathcal{S}^{N} \mid\right.$ for all $i \in N$ it holds that $\left.\left|S^{-1}(i)\right| \leq 1\right\}$ the class of all permission trees on $N$. For $T \subseteq N, T \neq \emptyset$, and $c_{T}>0$, the positively scaled unanimity game $w_{T}=c_{T} u_{T}$ is given by

$$
w_{T}(E)=\left\{\begin{array}{cl}
c_{T} & \text { if } T \subseteq E \\
0 & \text { otherwise }
\end{array}\right.
$$

For $S \in \mathcal{S}^{N}$ and $h \in N$, the permission structure $S_{-h}$ on $N \backslash\{h\}$ describes a permission structure without player $h$ where all successors of $h$ become successor of the predecessors of $h$, and thus is given by (Fig. 4)

$$
S_{-h}(i)= \begin{cases}(S(i) \backslash\{h\}) \cup S(h) & \text { if } i \in S^{-1}(h) \\ S(i) & \text { if } i \in N \backslash\left(\{h\} \cup S^{-1}(h)\right) .\end{cases}
$$

In the following we denote $\widehat{S}^{-1}(T)=\bigcup_{i \in T} \widehat{S}^{-1}(i)$ and $\bar{S}^{-1}(T)=\bigcup_{i \in T} \bar{S}^{-1}(i)$ for $T \subseteq N$.

Lemma 4.2 If solution $f$ satisfies power split neutrality, vertical split neutrality, horizontal split neutrality, one-player efficiency, the necessary player property and weak structural monotonicity, then $f\left(N, w_{T}, S\right)$ is uniquely determined for all $S \in \mathcal{S}_{\text {tree }}^{N}$ and $w_{T}=c_{T} u_{T}$ with $c_{T}>0$, and $T \subseteq N$ satisfying $T \cup \widehat{S}^{-1}(T)=N$.

Proof Suppose that solution $f$ satisfies the six axioms. Consider a hierarchical permission (tree) structure $S \in \mathcal{S}_{\text {tree }}^{N}$ and monotone characteristic function $w_{T}=c_{T} u_{T}, T \subseteq$ $N, c_{T}>0$ with $T \cup \widehat{S}^{-1}(T)=N$. (So, there are no inessential players.) Clearly, $\bar{f}$ satisfying the necessary player property implies that there exists a constant $c^{*} \in \mathbb{R}$ such that $f_{i}\left(N, w_{T}, S\right)=c^{*}$ for all $i \in T$, and $f_{i}\left(N, w_{T}, S\right) \leq c^{*}$ for all $i \in N \backslash T$. But then weak structural monotonicity and the fact that $\widehat{S}(i)=\overline{\bar{S}}(i)$ for all $i \in N$ and $S \in \mathcal{S}_{\text {tree }}^{N}$ imply that

$$
f_{i}\left(N, w_{T}, S\right)=c^{*} \text { for all } i \in T \cup \bar{S}^{-1}(T)=T \cup \widehat{S}^{-1}(T)=N .
$$

So, we have uniquely determined $f\left(N, w_{T}, S\right)$ if we determine $c^{*}$. We do this by induction on $|N|$.

If $|N|=1$ then one-player efficiency implies that $f_{i}\left(N, w_{T}, S\right)=c_{T}$ for $i \in N$. 
Fig. 5 An illustration of permission structures $S, S^{\prime}$ and $S^{\prime \prime}$ of the proof of Lemma 4.2 case (iii)
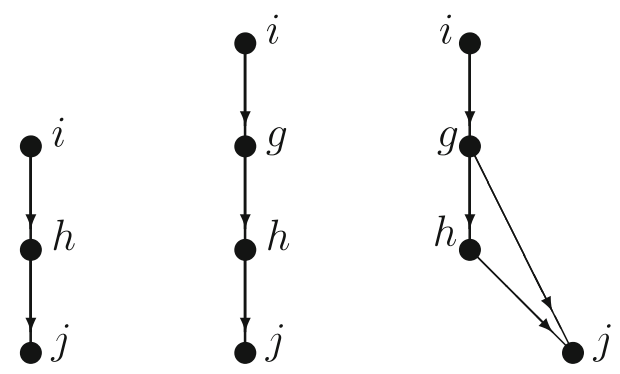

Proceeding by induction assume that $f_{i}\left(N^{\prime}, w_{T^{\prime}}, S^{\prime}\right)=\frac{c_{T^{\prime}}}{2^{\left|N^{\prime}\right|-1}}$ for all $\left(N^{\prime}, w_{T^{\prime}}, S^{\prime}\right)$ with $S^{\prime} \in \mathcal{S}_{\text {tree }}^{N^{\prime}}, c_{T^{\prime}}>0, T^{\prime} \cup\left(\widehat{S^{\prime}}\right)^{-1}\left(T^{\prime}\right)=N^{\prime}$ and $\left|N^{\prime}\right|<|N|$.

We distinguish the following three cases (of which at least one must occur).

(i) Suppose there exist $h, g, j \in N, h \neq g$, such that $S(h)=S(j)=\emptyset$ and $\{h, j\} \subseteq S(g)$. (Note that this case can only occur if $|N| \geq 3$.) By the assumption that $T \cup \widehat{S}^{-1}(T)=N$, we have $\{h, j\} \subseteq T$. Since $c_{T} u_{T}=\left(c_{T} u_{T \backslash\{h\}}\right)^{H(h, j)}$ and $S=\left(S_{-h}\right)^{H(h, j)}$ with $v^{H(h, j)}$ and $S^{H(h, j)}$ as given in (3) and (4), horizontal split neutrality implies that

$$
f_{j}\left(N, w_{T}, S\right)+f_{h}\left(N, w_{T}, S\right)=f_{j}\left(N \backslash\{h\}, c_{T} u_{T \backslash\{h\}}, S_{-h}\right) .
$$

Since $S_{-h} \in \mathcal{S}_{\text {tree }}^{N \backslash\{h\}}$ the induction hypothesis implies that $f_{j}(N \backslash\{h\}$, $\left.c_{T} u_{T \backslash\{h\}}, S_{-h}\right)=\frac{c_{T}}{2^{|N|-2}}$. With (7) and (8) it then follows that $2 c^{*}=\frac{c_{T}}{2^{|N|-2}}$, and thus $f_{i}\left(N, w_{T}, S\right)=c^{*}=\frac{c_{T}}{2^{|N|-1}}$ for all $i \in N$.

(ii) Suppose that there exist $j \in T$ and $h \in N \backslash T$ with $S(j)=\emptyset$ and $S(h)=\{j\}$. Since $w_{T}=\left(w_{T}\right)^{V(h, j)}$ and $S=\left(S_{-h}\right)^{V(h, j)}$ with $v^{V(h, j)}$ and $S^{V(h, j)}$ as given in (1) and (2), vertical split neutrality implies that

$$
f_{j}\left(N, w_{T}, S\right)+f_{h}\left(N, w_{T}, S\right)=f_{j}\left(N \backslash\{h\}, w_{T}, S_{-h}\right) .
$$

Similar as in case (i), with the induction hypothesis and (7) this yields that $2 c^{*}=\frac{c_{T}}{2^{|N|-2}}$, and thus $f_{i}\left(N, w_{T}, S\right)=c^{*}=\frac{c_{T}}{2^{|N|-1}}$ for all $i \in N$.

(iii) Suppose that there exist $h, j \in T$ with $S(j)=\emptyset$ and $S(h)=\{j\}$. Then take a $g \in \mathbb{N} \backslash N$, and define (see Fig. 5) $S^{\prime}, S^{\prime \prime} \in \mathcal{S}^{N \cup\{g\}}$ by $S^{\prime}=S^{V(g, h)}$ and

$$
S^{\prime \prime}(i)= \begin{cases}\{h, j\} & \text { if } i=g \\ (S(i) \backslash\{h\}) \cup\{g\} & \text { if } i \in S^{-1}(h) \\ S(i) & \text { if } i \in N \backslash S^{-1}(h)\end{cases}
$$

(Note that $S^{\prime \prime} \notin \mathcal{S}_{\text {tree }}^{N \cup\{g\}}$ since $h$ and $g$ are both predecessor of player $j$ in $S^{\prime \prime}$.) Since $T \cup{\overline{S^{\prime \prime}}}^{-1}(T)=N \cup\{g\}$, the necessary player property and weak structural monotonicity imply that there is a $c^{* *} \in \mathbb{R}$ such that

$$
f_{i}\left(N \cup\{g\}, w_{T}, S^{\prime \prime}\right)=c^{* *} \text { for all } i \in N \cup\{g\} .
$$


Since $\left((N \cup\{g\}) \backslash\{h\}, c_{T} u_{T \backslash\{h\}}, S_{-h}^{\prime \prime}\right) \in \mathcal{S}_{\text {tree }}^{(N \cup\{g\}) \backslash\{h\}}$ (with $\left.|(N \cup\{g\} \backslash\{h\})|=|N|\right)$ is as considered in case (ii), it follows from that case that

$$
f_{i}\left((N \cup\{g\}) \backslash\{h\}, c_{T} u_{T \backslash\{h\}}, S_{-h}^{\prime \prime}\right)=\frac{c_{T}}{2^{|N|-1}} \text { for all } i \in(N \cup\{g\}) \backslash\{h\} .
$$

But then, by $S_{-(h, j)}^{\prime \prime}=\left(S_{-h}^{\prime \prime}\right)^{H(h, j)}$ and $w_{T}=\left(c_{T} u_{T \backslash\{h\}}\right)^{H(h, j)}$, horizontal split neutrality implies that $f_{j}\left(N \cup\{g\}, w_{T}, S_{-(h, j)}^{\prime \prime}\right)+f_{h}\left(N \cup\{g\}, w_{T}, S_{-(h, j)}^{\prime \prime}\right)=$ $f_{j}\left((N \cup\{g\}) \backslash\{h\}, c_{T} u_{T \backslash\{h\}}, S_{-h}^{\prime \prime}\right)=\frac{c_{T}}{2^{|N|-1}}$. With the necessary player property, weak structural monotonicity and the fact that $T \cup \overline{S_{-(h, j)}^{\prime \prime}}-1 \quad(T)=N \cup\{g\}$ this yields

$$
f_{i}\left(N \cup\{g\}, w_{T}, S_{-(h, j)}^{\prime \prime}\right)=\frac{c_{T}}{2^{|N|}} \text { for all } i \in N \cup\{g\} .
$$

Further, power split neutrality implies that

$$
\begin{aligned}
f_{h}\left(N \cup\{g\}, w_{T}, S^{\prime \prime}\right)+f_{g}\left(N \cup\{g\}, w_{T}, S^{\prime \prime}\right)= & f_{h}\left(N \cup\{g\}, w_{T}, S_{-(h, j)}^{\prime \prime}\right) \\
& +f_{g}\left(N \cup\{g\}, w_{T}, S_{-(h, j)}^{\prime \prime}\right)
\end{aligned}
$$

which with (9) and (10) yields

$$
f_{i}\left(N \cup\{g\}, w_{T}, S^{\prime \prime}\right)=\frac{c_{T}}{2^{|N|}} \text { for all } i \in N \cup\{g\} .
$$

Since $S^{\prime}=S_{-(g, j)}^{\prime \prime}$ again applying power split neutrality yields

$$
\begin{aligned}
f_{h}\left(N \cup\{g\}, w_{T}, S^{\prime}\right)+f_{g}\left(N \cup\{g\}, w_{T}, S^{\prime}\right)= & f_{h}\left(N \cup\{g\}, w_{T}, S^{\prime \prime}\right) \\
& +f_{g}\left(N \cup\{g\}, w_{T}, S^{\prime \prime}\right)
\end{aligned}
$$

which with the necessary player property, weak structural monotonicity, $T \cup$ ${\overline{S^{\prime}}}^{-1}(T)=N \cup\{g\}$ and (12) yields

$$
f_{i}\left(N \cup\{g\}, w_{T}, S^{\prime}\right)=\frac{c_{T}}{2^{|N|}} \text { for all } i \in N \cup\{g\} .
$$

Finally, since $S^{\prime}=S^{V(g, h)}$ and $w_{T}=\left(w_{T}\right)^{V(g, h)}$, vertical split neutrality yields

$$
f_{h}\left(N, w_{T}, S\right)=f_{h}\left(N \cup\{g\}, w_{T}, S^{\prime}\right)+f_{g}\left(N \cup\{g\}, w_{T}, S^{\prime}\right)=\frac{c_{T}}{2^{|N|-1}},
$$

and thus with (7) we have $f_{i}\left(N, w_{T}, S\right)=c^{*}=\frac{c_{T}}{2^{|N|-1}}$ for all $i \in N$.

So, for $S \in \mathcal{S}_{\text {tree }}^{N}$ and $w_{T}=c_{T} u_{T}$ with $c_{T}>0$ and $T \cup \widehat{S}^{-1}(T)=N$, we conclude that $f_{i}\left(N, w_{T}, S\right)=c^{*}=\frac{c_{T}}{2^{|N|-1}}$ for all $i \in T \cup \widehat{S}^{-1}(T)=N$.

The next step is to show that adding the inessential player property and disjunctive fairness to the axioms implies that $f$ is uniquely determined for all positively scaled unanimity games with a permission tree structure (allowing inessential players). 
Lemma 4.3 If solution $f$ satisfies power split neutrality, vertical split neutrality, horizontal split neutrality, one-player efficiency, the inessential player property, the necessary player property, weak structural monotonicity and disjunctive fairness, then $f\left(N, w_{T}, S\right)$ is uniquely determined whenever $S \in \mathcal{S}_{\text {tree }}^{N}$ and $w_{T}=c_{T} u_{T}$ for some $T \subseteq N, T \neq \emptyset$, and $c_{T}>0$.

Proof Suppose that solution $f$ satisfies the eight axioms. Consider a permission (tree) structure $S \in \mathcal{S}_{\text {tree }}^{N}$ and monotone characteristic function $w_{T}=c_{T} u_{T}$ for some $c_{T}>0, T \subseteq N, T \neq \emptyset$. Denote $\alpha_{S}(T)=T \cup \widehat{S}^{-1}(T)$. We distinguish the case where all players, except the top-player, are inessential from the other cases.

A. We first consider the case that $T \neq\left\{i_{0}\right\}$.

Since all players in $N \backslash \alpha_{S}(T)$ are inessential players in $\left(N, w_{T}, S\right)$, the inessential player property implies that $f_{i}\left(N, w_{T}, S\right)=0$ for all $i \in N \backslash \alpha_{S}(T)$. Further, $f$ satisfying the necessary player property and weak structural monotonicity and the fact that $\widehat{S}(i)=\bar{S}(i)$ for all $i \in N$ and $S \in \mathcal{S}_{\text {tree }}^{N}$, imply that there exists a constant $c^{*} \in \mathbb{R}$ such that $f_{i}\left(N, w_{T}, S\right)=c^{*}$ for all $i \in \alpha_{S}(T)$. So,

$$
f_{i}\left(N, w_{T}, S\right)= \begin{cases}c^{*} & \text { if } i \in \alpha_{S}(T)=T \cup \widehat{S}^{-1}(T) \\ 0 & \text { if } i \in N \backslash \alpha_{S}(T) .\end{cases}
$$

So, we have determined $f\left(N, w_{T}, S\right)$ if we determine $c^{*}$. In particular, $f_{i_{0}}\left(N, w_{T}\right.$, $S)=c^{*}$. We determine $c^{*}$ by induction on $\left|N \backslash \alpha_{S}(T)\right|$. 4.2 .

If $\left|N \backslash \alpha_{S}(T)\right|=0$ then $c^{*}=\frac{c_{T}}{2^{|N|-1}}=\frac{c_{T}}{2^{\mid{ }^{2} S}(T) \mid-1}$ is uniquely determined by Lemma

Proceeding by induction assume that $c^{*}=f_{i_{0}}\left(N^{\prime}, w_{T^{\prime}}, S^{\prime}\right)$ is uniquely determined for all $\left(N^{\prime}, w_{T^{\prime}}, S^{\prime}\right)$ with $S^{\prime} \in \mathcal{S}_{\text {tree }}^{N^{\prime}}, c_{T^{\prime}}>0$ and $\left|N^{\prime} \backslash \alpha_{S^{\prime}}\left(T^{\prime}\right)\right|<\left|N \backslash \alpha_{S}(T)\right|$.

Since $N \backslash \alpha_{S}(T) \neq \emptyset$ there exists a $j \in N \backslash \alpha_{S}(T)$ with $S(j)=\emptyset$. We distinguish the following two cases (of which at least one must occur).

(i) Suppose that $\alpha_{S}(T) \neq\left\{i_{0}\right\}$ and $j \notin \widehat{S}(h)$ for all $h \in \alpha_{S}(T) \backslash\left\{i_{0}\right\}$. Since $T \neq\left\{i_{0}\right\}$ there is an $h \in \alpha_{S}(T) \cap S\left(i_{0}\right)$. Define $S^{\prime}, S^{\prime \prime} \in \mathcal{S}^{N}$ by

$$
S^{\prime}(i)= \begin{cases}\{h\} & \text { if } i=j \\ S(i) & \text { otherwise }\end{cases}
$$

and $S^{\prime \prime}=S_{-\left(i_{0}, h\right)}^{\prime}$. (Note that $S^{\prime} \notin \mathcal{S}_{\text {tree }}^{N}$ since player $h$ has two predecessors in $S^{\prime}$ (Fig. 6). Also note that $S^{\prime \prime} \in \mathcal{S}_{\text {tree }}^{N}$.)

Since $\left|N \backslash \alpha_{S^{\prime \prime}}(T)\right|<\left|N \backslash \alpha_{S}(T)\right|$, the induction hypothesis implies that all $f_{i}\left(N, w_{T}, S^{\prime \prime}\right), i \in N$, are known. Since $S=S_{-(j, h)}^{\prime}$ and $S^{\prime \prime}=S_{-\left(i_{0}, h\right)}^{\prime}$, twice applying power split neutrality implies that

$$
\begin{aligned}
f_{j}\left(N, w_{T}, S\right)+f_{i_{0}}\left(N, w_{T}, S\right) & =f_{j}\left(N, w_{T}, S^{\prime}\right)+f_{i_{0}}\left(N, w_{T}, S^{\prime}\right) \\
& =f_{j}\left(N, w_{T}, S^{\prime \prime}\right)+f_{i_{0}}\left(N, w_{T}, S^{\prime \prime}\right)
\end{aligned}
$$

which with (13) and the induction hypothesis yields that $f_{i}\left(N, w_{T}, S\right)=c^{*}, i \in$ $\alpha_{S}(T)$, is uniquely determined. 
Fig. 6 An illustration of permission structures $S^{\prime}$ and $S^{\prime \prime}$ of the proof of Lemma 4.3 case A.(i), with $j \in S\left(i_{0}\right)$
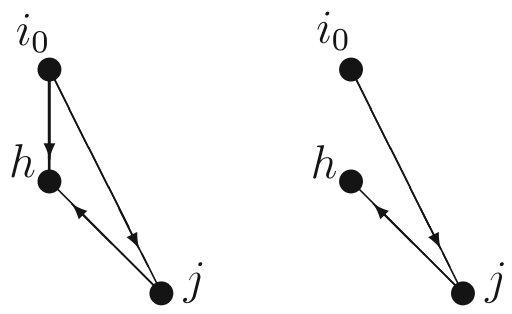

Fig. 7 An illustration of permission structures $S^{\prime}$ and $S^{\prime \prime}$ of the proof of Lemma 4.3 case A.(ii) with $h \in S\left(i_{0}\right)$ and $j \in S(h)$
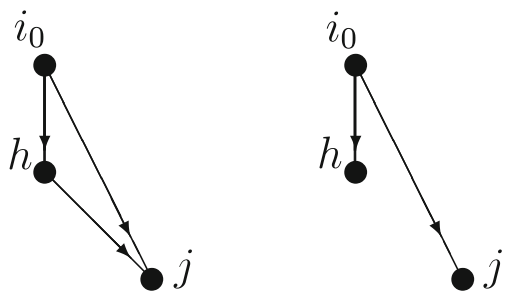

(ii) Suppose that $\alpha_{S}(T) \neq\left\{i_{0}\right\}$ and there is an $h \in \alpha_{S}(T) \backslash\left\{i_{0}\right\}$ with $j \in \widehat{S}(h)$. Define $S^{\prime}, S^{\prime \prime} \in \mathcal{S}^{N}$ by (Fig. 7)

$$
S^{\prime}(i)= \begin{cases}S(i) \cup\{j\} & \text { if } i=i_{0} \\ S(i) & \text { otherwise }\end{cases}
$$

and $S^{\prime \prime}=S_{-(g, j)}^{\prime}$ for $g \in S^{-1}(j)$ (possibly $g=h$ if $j \in S(h)$ ). (Note that $S^{\prime} \notin \mathcal{S}_{\text {tree }}^{N}$ since player $j$ has two predecessors in $S^{\prime}$. Also note that $S^{\prime \prime} \in \mathcal{S}_{\text {tree }}^{N}$.)

Since $S=S_{-\left(i_{0}, j\right)}^{\prime}$ and $j$ is an inessential player in $\left(N, w_{T}, S\right)$ and $\left(N, w_{T}, S^{\prime}\right)$, disjunctive fairness and the inessential player property imply that $f_{i_{0}}\left(N, w_{T}, S^{\prime}\right)-$ $f_{i_{0}}\left(N, w_{T}, S\right)=f_{j}\left(N, w_{T}, S^{\prime}\right)-f_{j}\left(N, w_{T}, S\right)=0$. Thus

$$
f_{i_{0}}\left(N, w_{T}, S^{\prime}\right)=f_{i_{0}}\left(N, w_{T}, S\right) .
$$

Since $S^{\prime \prime}=S_{-(g, j)}^{\prime}$ for $g \in S^{-1}(j)$, and $j$ is also an inessential player in $\left(N, w_{T}\right.$, $\left.S^{\prime \prime}\right)$, disjunctive fairness, the inessential player property and $g \in \overline{S^{\prime}}\left(i_{0}\right) \cap \overline{S^{\prime \prime}}\left(i_{0}\right)$ also imply that $f_{i_{0}}\left(N, w_{T}, S^{\prime}\right)-f_{i_{0}}\left(N, w_{T}, S^{\prime \prime}\right)=f_{j}\left(N, w_{T}, S^{\prime}\right)-f_{j}\left(N, w_{T}, S^{\prime \prime}\right)=0$. Thus $f_{i_{0}}\left(N, w_{T}, S^{\prime}\right)=f_{i_{0}}\left(N, w_{T}, S^{\prime \prime}\right)$. So, with (14) it follows that $f_{i_{0}}\left(N, w_{T}, S\right)=$ $f_{i_{0}}\left(N, w_{T}, S^{\prime}\right)=f_{i_{0}}\left(N, w_{T}, S^{\prime \prime}\right)$. Since $S^{\prime \prime}$ is as in case (i) we have uniquely determined $f_{i_{0}}\left(N, w_{T}, S\right)=f_{i_{0}}\left(N, w_{T}, S^{\prime \prime}\right)=c^{*}$.

So, in both cases A.(i) and A.(ii) we uniquely determined $c^{*}$ and thus, with (13) we uniquely determined $f\left(N, w_{T}, S\right)$.

B. Next we consider the case $T=\left\{i_{0}\right\}$.

Then $\alpha_{S}(T)=\left\{i_{0}\right\}$. The inessential player property implies that $f_{i}\left(N, w_{T}, S\right)=0$ for all $i \in N \backslash\left\{i_{0}\right\}$. 
Consider game with permission structure $\left(N \cup\{g\}, w_{T}, S^{V\left(g, i_{0}\right)}\right)$. Since $w_{T}=$ $\left(w_{T}\right)^{V\left(g, i_{0}\right)}$, vertical split neutrality implies that $f_{i_{0}}\left(N \cup\{g\}, w_{T}, S^{V\left(g, i_{0}\right)}\right)+$ $f_{g}\left(N \cup\{g\}, w_{T}, S^{V\left(g, i_{0}\right)}\right)=f_{i_{0}}\left(N, w_{T}, S\right)$. The necessary player property and weak structural monotonicity imply that $\left.f_{i_{0}}\left(N \cup\{g\}, w_{T}, S^{V\left(g, i_{0}\right)}\right)\right)=f_{g}(N \cup$ $\left.\left.\{g\}, w_{T}, S^{V\left(g, i_{0}\right)}\right)\right)$. Thus $\left.f_{i_{0}}\left(N, w_{T}, S\right)=2 f_{i_{0}}\left(N \cup\{g\}, w_{T}, S^{V\left(g, i_{0}\right)}\right)\right)$. Since $(N \cup$ $\left.\left.\{g\}, w_{T}, S^{V\left(g, i_{0}\right)}\right)\right)$ is as considered in Case A, we determined $f_{i_{0}}\left(N, w_{T}, S\right)=c^{*}=$ $\left.2 f_{i_{0}}\left(N \cup\{g\}, w_{T}, S^{V\left(g, i_{0}\right)}\right)\right)$.

Finally, by adding additivity to the axioms of Lemma 4.3 we prove the main result.

Proof of Theorem 4.1.(i) Since $\beta^{d}$ satisfying the nine axioms follows from Proposition 3.1 or follows similar as it is shown for Shapley permission values, we only show that there can be at most one solution that satisfies the nine axioms. Therefore, suppose that solution $f$ satisfies the nine axioms. Consider the hierarchical permission structure $S \in \mathcal{S}^{N}$ and the monotone characteristic function $w_{T}=c_{T} u_{T}, c_{T} \geq 0$, as given in (5). If $c_{T}=0$ then the inessential player property implies that $f_{i}\left(N, w_{T}, S\right)=0$ for all $i \in N$.

Now suppose that $c_{T}>0$. Again, we denote by $\alpha_{S}(T)=T \cup \widehat{S}^{-1}(T)$ the set consisting of all players in $T$ and all their superiors. For $S \in \mathcal{S}^{N} \backslash \mathcal{S}_{\text {tree }}^{N}$ there is at least one $i \in N$ with $\widehat{S}_{i}^{-1}(T) \neq \bar{S}_{i}^{-1}(T)$. Therefore, by $\gamma_{S}(T):=\left\{i \in \alpha_{S}(T)\right.$ | $T \cap(\{i\} \cup \bar{S}(i)) \neq \emptyset\}$ we denote the set of those players in $\alpha_{S}(T)$ who belong to $T$ or have subordinates in $T$ that they dominate completely.

Again, the inessential player property implies that $f_{i}\left(N, w_{T}, S\right)=0$ for all $i \in N \backslash \alpha_{S}(T)$. Further, $f$ satisfying the necessary player property and weak structural monotonicity implies that there exists a constant $c^{*} \in \mathbb{R}$ such that $f_{i}\left(N, w_{T}, S\right)=c^{*}$ for all $i \in \gamma_{S}(T)$. We prove that $c^{*}$ and all $f_{i}\left(N, w_{T}, S\right), i \in \alpha_{S}(T) \backslash \gamma_{S}(T)$, are uniquely determined by induction on the number $\sum_{i \in N}|S(i)|$. (Note that $\sum_{i \in N}|S(i)| \geq|N|-1$ for all $S \in \mathcal{S}^{N}$.)

If $\sum_{i \in N}|S(i)|=|N|-1$ then $S \in \mathcal{S}_{\text {tree }}^{N}$ and $f\left(N, w_{T}, S\right)$ is uniquely determined by Lemma 4.3.

Proceeding by induction assume that $f\left(N, w_{T}, S^{\prime}\right)$ is uniquely determined for all $S^{\prime} \in \mathcal{S}^{N}$ with $\sum_{i \in N}\left|S^{\prime}(i)\right|<\sum_{i \in N}|S(i)|$.

Next we recursively define the sets $L_{k}, k \in\{0\} \cup \mathbb{N}$, by

$$
\begin{aligned}
& L_{0}:=\emptyset, \text { and } \\
& L_{k}:=\left\{i \in N \backslash \bigcup_{t=1}^{k-1} L_{t} \mid S(i) \subseteq \bigcup_{t=1}^{k-1} L_{t}\right\} \text {, for all } k \in \mathbb{N} .
\end{aligned}
$$

In van den Brink and Gilles (1994) it is shown that for hierarchical permission structures there exists an $M<\infty$ such that the sets $L_{1}, \ldots, L_{M}$ form a partition of $N$ consisting of non-empty sets only.

Next we describe a procedure which determines the values $f_{i}\left(N, w_{T}, S\right)$ as $c^{*}$ plus some constant $c_{i}$, i.e. we determine the values $c_{i}, i \in N$, such that

$$
f_{i}\left(N, w_{T}, S\right)=c^{*}+c_{i}, \text { for all } i \in N
$$


Step 1 For every $i \in L_{1}$ one of the following two conditions is satisfied:

(i) If $i \in N \backslash \alpha_{S}(T)$ then $f_{i}\left(N, w_{T}, S\right)=0$ as mentioned before. Thus $c_{i}=-c^{*}$.

(ii) If $i \in \alpha_{S}(T)$ then $i \in T$ since $S(i)=\emptyset$. Thus $f_{i}\left(N, w_{T}, S\right)=c^{*}$, i.e. $c_{i}=0$.

Set $k=2$. GoTO STEP 2 .

Step 2 If $L_{k}=\varnothing$ then STOP.

Else, for every $i \in L_{k}$ one of the following three conditions is satisfied:

(i) If $i \in N \backslash \alpha_{S}(T)$ then $f_{i}\left(N, w_{T}, S\right)=0$, and thus $c_{i}=-c^{*}$.

(ii) If $i \in \gamma_{S}(T)$ then $f_{i}\left(N, w_{T}, S\right)=c^{*}$, and thus $c_{i}=0$.

(iii) If $i \in \alpha_{S}(T) \backslash \gamma_{S}(T)$ then by definition of $\alpha_{S}(T)$ and $\gamma_{S}(T)$ there exists an $h \in\{i\} \cup \bar{S}(i)$ and a $j \in S(h)$ such that $\left|S^{-1}(j)\right| \geq 2$. Disjunctive fairness implies that

$$
\begin{aligned}
& f_{i}\left(N, w_{T}, S\right)-f_{i}\left(N, w_{T}, S_{-(h, j)}\right) \\
& \quad=f_{j}\left(N, w_{T}, S\right)-f_{j}\left(N, w_{T}, S_{-(h, j)}\right),
\end{aligned}
$$

and thus

$$
c_{i}=c_{j}-f_{j}\left(N, w_{T}, S_{-(h, j)}\right)+f_{i}\left(N, w_{T}, S_{-(h, j)}\right) .
$$

Using the induction hypothesis (implying that $f_{j}\left(N, w_{T}, S_{-(h, j)}\right)$ and $f_{i}\left(N, w_{T}, S_{-(h, j)}\right)$ are known), and the fact that $j \in \widehat{S}(i)$ (implying that we already determined $c_{j}$ since $j \in L_{l}$ with $l<k$ ), it follows that we have determined $c_{i}$.

\section{Step 3 Set $k=k+1$. GoTO STEP 2.}

Since there exists an $M<\infty$ such that the sets $L_{1}, \ldots, L_{M}$ form a partition of $N$ consisting of non-empty sets only, the procedure described above determines the values $c_{i}, i \in N$, which with (15) yield $f_{i}\left(N, w_{T}, S\right)=c^{*}+c_{i}$ for all $i \in N$. So, we only have to determine $c^{*}$. We distinguish the following two cases.

(i) Suppose there exists $j \in \alpha_{S}(T)$ with $\left|S^{-1}(j)\right| \geq 2$. Take $h, g \in N, h \neq g$, such that $j \in S(h) \cap S(g)$. Power split neutrality implies that

$$
f_{h}\left(N, w_{T}, S\right)+f_{g}\left(N, w_{T}, S\right)=f_{h}\left(N, w_{T}, S_{-(g, j)}\right)+f_{g}\left(N, w_{T}, S_{-(g, j)}\right),
$$

which with the induction hypothesis yields that $f_{h}\left(N, w_{T}, S\right)+f_{g}\left(N, w_{T}, S\right)$ is known. With (15) and the fact that all $c_{i}$ are known, $c^{*}$ is uniquely determined.

(ii) Suppose that $\left|S^{-1}(j)\right|=1$ for all $j \in \alpha_{S}(T) \backslash\left\{i_{0}\right\}$. Then, by assumption, there exists a $j \in N \backslash \alpha_{S}(T)$ with $\left|S^{-1}(j)\right| \geq 2$. Take $h \in S^{-1}(j)$. Disjunctive fairness, the inessential player property and $j \in \bar{S}\left(i_{0}\right)$ imply that

$$
f_{i_{0}}\left(N, w_{T}, S\right)-f_{i_{0}}\left(N, w_{T}, S_{-(h, j)}\right)=f_{j}\left(N, w_{T}, S\right)-f_{j}\left(N, w_{T}, S_{-(h, j)}\right)=0 .
$$

So, $c^{*}=f_{i_{0}}\left(N, w_{T}, S\right)=f_{i_{0}}\left(N, w_{T}, S_{-(h, j)}\right)$ is uniquely determined by the induction hypothesis. 
In both cases we determined $c^{*}$. Since we already determined all $c_{i}, i \in N$, with (15) we then uniquely determined all values $f_{i}\left(N, w_{T}, S\right), i \in N$, for $w_{T}=$ $c_{T} u_{T}, c_{T} \geq 0$.

Now, let $S \in \mathcal{S}^{N}$ and consider the characteristic function $w_{T}=c_{T} u_{T}$ with $c_{T}<0 .{ }^{10}$ Let $v_{0} \in \mathcal{G}^{N}$ denote the null game, i.e., $v_{0}(E)=0$ for all $E \subseteq N$. From the inessential player property it follows that $f_{i}\left(N, v_{0}, S\right)=0$ for all $i \in N$. Since $w_{T}+\left(-w_{T}\right)=v_{0}$, additivity of $f$ implies that $f\left(N, w_{T}, S\right)=$ $f\left(N, v_{0}, S\right)-f\left(N,-w_{T}, S\right)=-f\left(N,-w_{T}, S\right)$. Since $-w_{T}=-c_{T} u_{T}$ with $-c_{T}>$ 0 is monotone, $f\left(N,-w_{T}, S\right)$ is uniquely determined. Thus also $f\left(N, w_{T}, S\right)=$ $-f\left(N,-w_{T}, S\right)$ is uniquely determined if $c_{T}<0$.

Finally, since every characteristic function $v \in \mathcal{G}^{N}$ can be expressed as a linear combination of unanimity games as $v=\sum_{\substack{T \subseteq N \\ T \neq \emptyset}} \Delta_{(N, v)}(T) u_{T}$ with $\Delta_{(N, v)}(T)=$ $\sum_{F \subseteq T}(-1)^{|T|-|F|} v(F)$ being the Harsanyi dividends (see Harsanyi 1959), it follows with additivity of $f$ that $f(N, v, S)$ is uniquely determined for every $v \in \mathcal{G}^{N}$ and $S \in \mathcal{S}^{N}$.

The proof of Theorem 4.1.(ii) is obtained in a similar way and is therefore omitted. We end this section by showing logical independence of the nine axioms of Theorem 4.1.(i). In each of the paragraphs below, we indicate which of the axioms of Theorem 4.1.(i) the solution defined in the paragraph violates:

1. The solution $f$ given by $f_{i_{0}}(N, v, S)=v\left(\left\{i_{0}\right\}\right)$, and $f_{i}(N, v, S)=0$ for all $i \in N \backslash\left\{i_{0}\right\}$ does not satisfy vertical split neutrality. (Remember that $i_{0}$ denotes the top-player in $S$.)

2. The solution $f$ given by $f(N, v, S)=\beta^{d}\left(N, \sum_{i \in N} v(\{i\}) u_{\{i\}}, S\right)$ does not satisfy horizontal split neutrality.

3. For $S \in \mathcal{S}^{N}$ and $T \subseteq N$ let $\mathcal{Z}_{T}^{S}=\left\{Z \in \mathcal{S}^{N} \mid Z^{-1}(i)=S^{-1}(i)\right.$ for all $i \in N \backslash \alpha_{S}(T), Z^{-1}(i) \subseteq S^{-1}(i)$ for all $i \in \alpha_{S}(T)$, and $\left|Z^{-1}(i)\right|=1$ for all $\left.i \in \alpha_{S}(T) \backslash\left\{i_{0}\right\}\right\}$ where again $\alpha_{S}(T)=T \cup \widehat{S}^{-1}(T)$. The solution $f$ given by $f(N, v, S)=\sum_{T \subseteq N} \sum_{Z \in \mathcal{Z}_{T}^{S}} \beta_{i}^{d}\left(N, \Delta_{(N, v)}(T) u_{T}, Z\right)$, where $\Delta_{(N, v)}(T), T \subseteq$ $N, T \neq \emptyset$, are the Harsanyi dividends, does not satisfy power split neutrality.

4. The solution $f$ given by $f_{i}(N, v, S)=0$ for all $i \in N$ does not satisfy one-player efficiency.

5. For game $(N, v)$ define $d(N, v)=\max \left\{\Delta_{(N, v)}(T) \mid T \subseteq N\right\}$ and $D(N, v)=$ $\left\{T \subseteq N \mid \Delta_{(N, v)}(T)=d(N, v)\right\}$. The solution $f$ given by $f(N, v, S)=$ $\beta^{d}\left(\bar{N}, \sum_{T \in D(N, v)} \Delta_{(N, v)}(T) u_{T}, S\right)$ does not satisfy additivity.

6. The solution $f$ given by $f_{i}(N, v, S)=\frac{v(N)}{2^{|N|-1}}$ for all $i \in N$, does not satisfy the inessential player property.

7. The solution $f$ given by $f_{i_{0}}(N, v, S)=v(N)$ and $f_{i}(N, v, S)=0$ for all $i \in$ $N \backslash\left\{i_{0}\right\}$ does not satisfy the necessary player property.

8. The solution $f$ given by $f(N, v, S)=B a(N, v)$ does not satisfy weak structural monotonicity.

9. The conjunctive Banzhaf permission value $\beta^{c}$ does not satisfy disjunctive fairness.

\footnotetext{
10 Note that we cannot apply the necessary player property and weak structural monotonicity to this game with permission structure, since $c_{T} u_{T}$ is not monotone if $c_{T}<0$.
} 
Table 1 Fairness and split neutrality properties of permission values

\begin{tabular}{lll}
\hline & Split neutrality & Grand split neutrality \\
\hline Disjunctive fairness & $\beta^{d}$ & $\varphi^{d}$ \\
Conjunctive fairness & $\beta^{c}$ & $\varphi^{c}$ \\
\hline
\end{tabular}

Logical independence of the axioms of Theorem 4.1.(ii) can be shown by the above solutions $1,4,6,7$ and 8 , using $\beta^{c}$ instead of $\beta^{d}$ in solutions 2, 3 and 5, and replacing $\beta^{c}$ by $\beta^{d}$ in solution 9 .

\section{Concluding remarks}

In this paper we gave axiomatic characterizations of the disjunctive- and conjunctive Banzhaf permission values. Comparing these axiomatizations with known axiomatizations of the Shapley permission values it turns out that efficiency of the Shapley permission values is replaced by four other axioms: power split neutrality, vertical split neutrality, horizontal split neutrality and one-player efficiency. Instead of using efficiency, we can also characterize the Shapley permission values by similar split neutrality axioms as used in characterizing the Banzhaf permission values, but requiring that splitting a player in two does not change the total sum of payoffs that is distributed among all players (instead of the sum of the payoffs of two players that are involved). Since the worth that is generated by the 'grand coalition' $N$, respectively $N \cup\{h\}$, does not change after a power- or player split, for any efficient solution the sum of payoffs over all players should not change. Adapting power split neutrality, vertical split neutrality and horizontal split neutrality in this way, it can be shown that replacing these axioms in Theorem 4.1 by the three 'grand' versions described above yields new characterizations of the two Shapley permission values. Comparing these new axiomatizations with those stated in Theorem 2.3, there are two advantages of these new axiomatizations. First, the new characterization uses weaker axioms since efficiency of a solution implies grand power split neutrality, grand vertical split neutrality, grand horizontal split neutrality and one-player efficiency, but the reverse does not hold. Second, the new axioms are comparable with the axioms characterizing the Banzhaf permission values in Theorem 4.1 in the sense that they only differ in using the 'grand' or 'pairwise' power and player split neutrality properties. According to these characterizations, the difference between the Banzhaf- and Shapley permission values is with respect to how the payoffs react to particular changes in the game and permission structure that reflect a split of power and/or players. The main difference between the disjunctive- and conjunctive permission values is with respect to the fairness axiom that is used. These differences are summarized in Table 1.

Although we showed that the Shapley permission values do not satisfy the opposite change property, they do satisfy this property for monotone games. More specific, Table 2 summarizes the effects of deleting the arc between players $h$ and $j \in S(h)$ on the payoffs of players $j, h$ and $g \in S^{-1}(j) \backslash\{h\}$ for monotone games with a permission structure. Note that power split neutrality as well as the two fairness axioms consider situations in which an arc is deleted from the permission structure. The properties stated 
Table 2 Effect on permission values of deleting arc $(h, j)$ with $j \in S(g), g \neq h$

\begin{tabular}{lll}
\hline $\begin{array}{l}\text { Effect on payoff } \\
\text { of player }\end{array}$ & $\begin{array}{l}\text { Disjunctive } \\
\text { approach }\end{array}$ & $\begin{array}{l}\text { Conjunctive } \\
\text { approach }\end{array}$ \\
\hline$h$ & - & - \\
$g$ & + & + \\
$j$ & - & + \\
\hline
\end{tabular}

in Table 2 thus can be seen as stability properties in line with Myerson (1977)'s stability notion for games with a communication (graph) structure. For monotone games with a permission structure, deleting an arc between two players never increases the Banzhafand Shapley permission values for the predecessor on the arc. This expresses that in monotone games it never hurts to dominate other players. For the other predecessors of the successor on the deleted arc, the permission values never decrease after deleting this arc. This expresses that in such games it never hurts to dominate your successors with as few other players as possible. For the successor on the arc there is a distinction between the disjunctive- and conjunctive permission values. After deleting an arc with one of its predecessors, its disjunctive permission value does not increase (because having more predecessors increases the cooperation possibilities of a player in the disjunctive approach), while its conjunctive permission value does not decrease (because having more predecessors restricts the cooperation possibilities of a player in the conjunctive approach).

Note that, although the axioms of Lemma 4.3 determine the solution outcomes for games with a permission tree, we did not characterize $\beta^{d}$ on the class of games with a permission tree since in the proof we used twice (in every induction step) permission structures that are not trees. In order to characterize the Banzhaf permission values on the class of games with a permission tree we need to avoid the graph manipulations yielding the structures $S^{\prime}$ in the Cases A.(i) and A.(ii), for example, by using an axiom which states that inessential players can be 'deleted' from the game and permission structure without changing the payoffs of other players. ${ }^{11}$

Acknowledgment I would like to thank two anonymous referees and the editor for useful remarks on a previous draft.

Open Access This article is distributed under the terms of the Creative Commons Attribution Noncommercial License which permits any noncommercial use, distribution, and reproduction in any medium, provided the original author(s) and source are credited.

\footnotetext{
11 This is a generalization of the null player out property for TU-games (see Derks and Haller 1999). A generalization of this property is used in Algaba et al. (2004) to characterize the Banzhaf value for games on antimatroids which generalizes the necessity of the axioms in Theorem 4.1. From the proofs of Theorem 2.3 given in van den Brink $(1997,1999)$ it follows that those axioms (even without the corresponding fairness) do characterize the Shapley permission values restricted to the class of games with a permission tree.
} 


\section{Appendix: A split neutrality of the Banzhaf value}

In proving that the Banzhaf permission values satisfy vertical- and horizontal split neutrality we use the following property of the Banzhaf value for TU-games which is similar as collusion neutrality defined in Haller (1994) to characterize the Banzhaf value for TU-games. This proposition states that if a player $h \in \mathbb{N} \backslash N$ enters the game $v \in \mathcal{G}^{N}$ as a veto player for player $j \in N$, then the sum of the Banzhaf values of players $h$ and $j$ in the new game is equal to the Banzhaf value of player $j$ in the original game. The straightforward proof is omitted. ${ }^{12}$

Proposition A.1 (On TU-games) If $N \subset \mathbb{N}, v \in \mathcal{G}^{N}, j \in N$ and $h \in \mathbb{N} \backslash N$ then

$$
B a_{h}\left(N \cup\{h\}, v_{h j}\right)+B a_{j}\left(N \cup\{h\}, v_{h j}\right)=B a_{j}(N, v),
$$

where $v_{h j} \in \mathcal{G}^{N \cup\{h\}}$ is given by

$$
v_{h j}(E)= \begin{cases}v(E \backslash\{j\}) & \text { if } E \subseteq N \\ v(E \cap N) & \text { if } E \subseteq N \cup\{h\}, \quad h \in E .\end{cases}
$$

\section{References}

Algaba E, Bilbao JM, van den Brink R, Jiménez-Losada A (2004) An axiomatizations of the Banzhaf value for cooperative games on antimatroids. Math Methods Oper Res 59:147-166

Aumann RJ, Drèze JH (1974) Cooperative Games with Coalition Structure. Int J Game Theory 3:217-237 Banzhaf JF (1965) Weighted voting doesn't work: a mathematical analysis. Rutgers Law Rev 19:317-343 Derks JJM, Haller HH (1999) Null players out? linear values for games with variable supports. Int Game Theory Rev 1:301-314

Dubey P, Shapley LS (1979) Mathematical properties of the Banzhaf power index. Math Oper Res 4:99-131

Gilles RP, Owen G (1999) Cooperative games and disjunctive permission structures. CentER discussion paper 9920, CentER for Economic Research. Tilburg University, Tilburg

Gilles RP, Owen G, van den Brink R (1992) Games with permission structures: the conjunctive approach. Int J Game Theory 20:277-293

Haller H (1994) Collusion properties of values. Int J Game Theory 23:261-281

Harsanyi JC (1959) A bargaining model for cooperative $n$-person games. In: Tucker AW, Luce RD (eds) Contributions to the theory of games IV. Princeton University Press, Princeton pp 325-355

Lehrer E (1988) An axiomatization of the Banzhaf value. Int J Game Theory 17:89-99

Myerson RB (1977) Graphs and cooperation in games. Math Oper Res 2:225-229

Owen G (1975) Multilinear extensions and the Banzhaf value. Nav Res Logist Q 22:741-750

Shapley LS (1953) A value for $n$-person games. In: Kuhn HW, Tucker AW (eds) Annals of Mathematics Studies 28 (Contributions to the Theory of Games Vol. 2). Princeton UP, Princeton pp 307-317

van den Brink R (1997) An axiomatization of the disjunctive permission value for games with a permission structure. Int J Game Theory 26:27-43

van den Brink R (1999) An axiomatization of the conjunctive permission value for games with a hierarchical permission structure. In: de Swart H (ed) Logic, game theory and social choice. Springer, Berlin, pp 125-139

\footnotetext{
12 Refering to this property as split neutrality for TU-games, it can be verified along the same lines as a similar result shown in Haller (1994), that the Banzhaf value for TU-games is characterized by one-player efficiency, the null player property, symmetry, additivity and split neutrality. Replacing split neutrality by a 'grand' version stating that the sum of the payoffs of all players does not change yields an axiomatization of the Shapley value.
} 
van den Brink R, Gilles RP (1994) A social power index for hierarchically structured populations of economic agents. In: Gilles RP, Ruys PHM (eds) Imperfections and behaviour in economic organizations. Kluwer, Dordrecht pp 279-318

van den Brink R, Gilles RP (1996) Axiomatizations of the conjunctive permission value for games with permission structures. Games Econ Behav 12:113-126 